\title{
Rursus
}

Russus

Poiétique, réception et réécriture des textes antiques

$7 \mid 2012$

L'encyclopédie zoologique de Constantin VII

\section{Die Textgeschichte der Historia Animalium des Aristoteles, Aristophanes von Byzanz und die zoologische Sylloge des Konstantinos Porphyrogennetos}

The Textual History of Aristotle's Historia Animalium, Aristophanes of Byzantium and the Zoological Sylloge of Constantine Porphyrogenitus

\section{Friederike Berger}

\section{OpenEdition \\ Journals}

Édition électronique

URL : http://journals.openedition.org/rursus/766

DOI : $10.4000 /$ rursus.766

ISSN : 1951-669X

Éditeur

Université Nice-Sophia Antipolis

Édition imprimée

Date de publication : 2 avril 2012

Référence électronique

Friederike Berger, «Die Textgeschichte der Historia Animalium des Aristoteles, Aristophanes von

Byzanz und die zoologische Sylloge des Konstantinos Porphyrogennetos », Rursus [En ligne], 7 | 2012, mis en ligne le 25 avril 2012, consulté le 01 mai 2019. URL : http://journals.openedition.org/ rursus/766; DOI : 10.4000/rursus.766

Ce document a été généré automatiquement le 1 mai 2019.

Rursus 


\section{Die Textgeschichte der Historia Animalium des Aristoteles, Aristophanes von Byzanz und die zoologische Sylloge des Konstantinos Porphyrogennetos}

The Textual History of Aristotle's Historia Animalium, Aristophanes of Byzantium and the Zoological Sylloge of Constantine Porphyrogenitus

Friederike Berger

In dem Augenblick, in dem ein Autor sein Werk abschließt und es der Öffentlichkeit übergibt, löst es sich von ihm. Es wird von fremden Augen gelesen, wird annotiert, kommentiert, exzerpiert. Sein Inhalt taucht in neuen Zusammenhängen auf, wird falsch verstanden oder verfälscht, gekürzt oder ergänzt und endlich ediert. Es ist Aufgabe des Editors, die Geschichte des Textes zu verstehen. Er muss dabei die Primär- und Sekundärüberlieferung sowie die Wirkungsgeschichte des Textes berücksichtigen und wird durch seine Edition schließlich selbst Teil der Textgeschichte werden. Der Vorgang ist schon kompliziert, wenn es um die Textgestaltung eines Werkes geht, er wird komplizierter, wenn es sich wie bei dem uns interessierenden Gegenstand um mehrere Werke und Bearbeitungsphasen handelt. In der Mitte des 4.Jh. v. Chr. verfasste Aristoteles seine tierkundlichen Werke. Da diese nicht als Nachschlagewerk für einzelne Tiere gedacht waren, lag es nahe, auf ihrer Grundlage ein solches zu konzipieren ${ }^{1}$. So bearbeitete 150 Jahre später Aristophanes von Byzanz Aristoteles' Werk in einer sogenannten Epitome, im 10. Jh. n. Chr. schließlich wird diese Epitome Teil einer Sylloge, deren Verfasser wiederum versucht, möglichst viele Texte über die Lebewesen zusammenzutragen. 


\section{Die handschriftliche Überlieferung}

2 Nur die Werke des Aristoteles selbst sind vollständig durch handschriftliche Überlieferung auf uns gekommen. Moderne Editionen der Historia Animalium können sich auf 25 Handschriften stützen, von denen 13 texttragend sind, d.h. keine bekannte Vorlage haben, von der sie direkt abstammen ${ }^{2}$. Einige dieser Handschriften überliefern den Text nicht vollständig oder sind nicht mehr vollständig erhalten. Das gilt insbesondere für ein Fragment aus dem 9.Jh.im Parisinus Suppl. gr.1156, das ursprünglich zum berühmten Aristoteles-Codex Vindob. phil. 100 gehörte. Diese Handschrift, die sicher in Konstantinopel im Kreis um den Patriarchen Photios geschrieben wurde, war später im Besitz von Wilhelm von Moerbeke, der sie für seine Übersetzung aristotelischer Werke heranzog (vUiLLEmIN-DIEM, 1982: $167 \mathrm{ff}$; anders BEULLENS-BOSSIER, 2000: LXXVIII, vgl. jedoch BERGER, 2005: 182-186.). Mit dieser Übersetzung ist also eine Textfassung der Historia Animalium aus dem 9. Jh. für uns greifbar. Im 9. Jh. ist auch die arabische Übersetzung entstanden. Man kann wohl annehmen, dass ihre Vorlage aus derselben Zeit stammt (vgl. BERGER, 2005: 52-54 mit weiterer Literatur).

Aus den erhaltenen Textzeugen lassen sich für den byzantinischen Raum zwei Versionen des Werkes rekonstruieren: der Hyparchetypus $\varphi$, der die Bücher I-IX überlieferte, und der Hyparchetypus $\chi$, der auch das zehnte Buch enthielt. Darüber hinaus ist es vielleicht bemerkenswert, dass der Hyparchetypus $\gamma$ möglicherweise ursprünglich nur die ersten sieben Bücher enthielt (I-VI, VIII), so dass er für Buch VIII (IX) und IX (VII) seine Vorlage wechseln musste. Gerade diese Bücher gelten neben dem nur im Vat.gr. $262\left(D^{a}\right)$ überlieferten Buch X in der heutigen Forschung als korrupt. Unsere Forschungen zeigen, dass schon BEKKER (1831) und DITTMEYER (1907) mit dem Marc. gr. 208 (A $\left.{ }^{a}\right)$, dem Laur. 87, $4\left(C^{a}\right)$ und dem Vat. gr. $262\left(D^{a}\right)$ für ihre Editionen im Prinzip die richtigen Handschriften herangezogen haben (A, C, D bei BERGER, 2005). Für die ersten Bücher kann mit dem Vat. Palat. gr. $260\left(\mathrm{Y}^{\mathrm{c}}\right)$ ein weiterer Textzeuge gewonnen werden. Nur der Vaticanus 1339 (P) wurde in seiner Bedeutung überschätzt, hier müssen die anderen texttragenden Handschriften der Familie ymit herangezogen werden. Leider sind diese Verhältnisse in der Neuedition von Balmes sehr unklar dargestellt, da hier die Lesarten aller Apographa von $\mathrm{A}^{\text {a }}$ mit im kritischen Apparat angegeben wurden (Balme, 2002).

Patriarch Photios, den wir mit dem ältesten Textzeugen der Historia Animalium in Verbindung brachten, schrieb im Codex 161 seiner Bibliothek, dass er Auszüge aus dem ersten und zweiten Buch der Epitome gelesen habe, und zwar in den Eklogai des Sophisten Sopatros, der im 4. Jh. n. Chr gelebt haben soll. Im elften Buch der Eklogai stehen diese Exzerpte zwischen Texten aus Plutarch und einer Theatergeschichte:

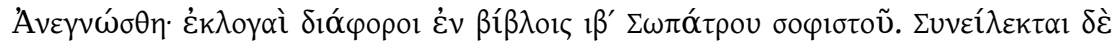

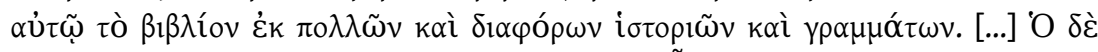

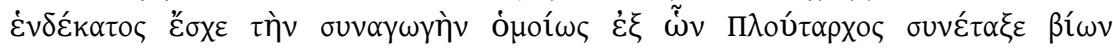

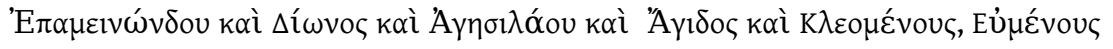

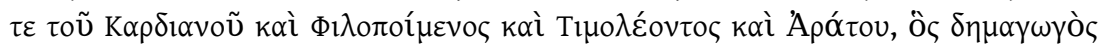

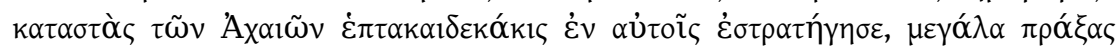

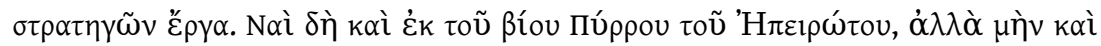

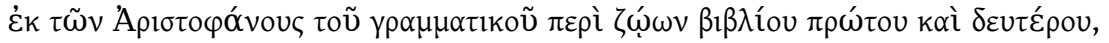

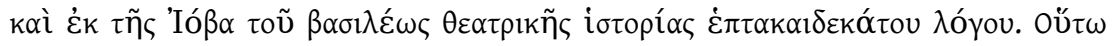

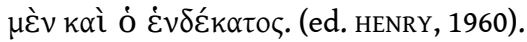



Handschriften der Historia Animalium stammen. Leider ist der Schreiber der Handschrift nicht bekannt, doch machte Valentina Cuomo jüngst auf ihren Besitzer aufmerksam (Cuomo, 2005): Es handelt sich um Theodosios Prinkeps, der von 1278 bis 1283 Patriarch von Antiocheia war, aber wohl in Konstantinopel residierte. Dort ist zu dieser Zeit ein gewisses Interesse an den naturwissenschaftlichen Werken des Aristoteles nachweisbar: Georgios Pachymeres (1242-1310) behandelt in seiner Philosophia (Pappa, 2009) De Generatione Animalium und De Partibus Animalium, Theodoros Metochites kommentierte diese Werke etwas später, von seinem Schüler Nikephoros Gregoras (BERGER, 2005: 162-163) kennen wir Exzerpte aus der Historia Animalium. Im 19. Jh. kam ein Teil der Handschrift in die Bibliothèque Nationale nach Paris, so dass das erste Buch nun im Paris. suppl gr. 495 erhalten ist, während sich das zweite in der ursprünglichen Handschrift Dionysiu 180 auf dem Athos befindet. Weitere Handschriften enthalten vor allen Dingen Exzerpte aus dem ersten Buch, so der Paris. gr. 1921, der im letzten Viertel des 14. Jh. in Konstantinopel entstanden ist und auch den Text der Historia Animalium überliefert. Der Schreiber dieser Handschrift ist uns aus zahlreichen Aristoteles-Handschriften bekannt und wird daher als Anonymus Aristotelicus bezeichnet (MONDRAIN, 2000: 11-33). Etwas umfangreicher ist eine Sammlung im Laur. 86, 8 aus dem 15. Jh. ${ }^{5}$

Der Text der Epitome des Aristophanes lässt sich nicht in das Stemma der Historia Animalium einbinden (BERGER, 2005: 201). Die aristotelischen Texte werden so frei wiedergegeben, dass kaum Lesarten zu gewinnen sind.

\section{Die Quellen der Epitome}

Hauptquelle für die Epitome sind zweifellos die aristotelischen Tierschriften, insbesondere die Historia Animalium. Da Aristophanes diese Werke vor der Edition durch Andronikos von Rhodos im ersten vorchristlichen Jahrhundert nutzte, ist es von Bedeutung, in welcher Form er die Texte vorfand. Eine bei Diogenes Laertios wiedergegebene Werkliste aus Hermippos führt ins 3. Jh. v. Chr. und damit in die Zeit, in der Aristophanes wirkte ( BERGER, 2005: 201):

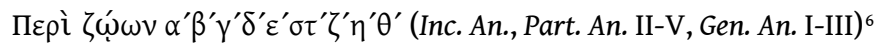

Av $\alpha \tau 0 \mu \tilde{\omega} v \alpha^{\prime} \beta^{\prime} \gamma^{\prime} \delta^{\prime} \varepsilon^{\prime} \sigma \tau^{\prime} \zeta^{\prime}$ (Anatomai + Hist. An. I-V, VI/VII, VIII)

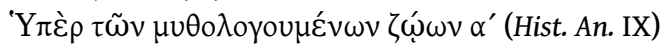

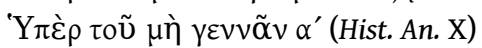

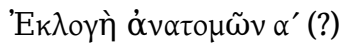


'Y $\pi \dot{\varepsilon} \rho \tau \tilde{\omega} v \sigma u v \theta \varepsilon \dot{\tau} \tau \omega \nu \zeta \hat{\omega} \omega \nu \alpha^{\prime}$ (Part. An. I ?)

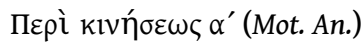

Das sechste und siebte Buch der Historia Animalium bildeten eine Einheit, die erst bei der Neuordnung aufgelöst wurde. Damals wurden wohl auch das heutige neunte und zehnte Buch hinzugefügt. Zu den ersten sechs Büchern der Historia Animalium gehörten die heute verlorenen Anatomai als Tafelwerk (vgl. auch STÜCKELBERGER, 1994: 74-78; Stückelberger, 1993: 139 ff.; Hellmann, 2004: 65-86), weshalb die beiden Werke bei Hermippos unter diesem Titel zitiert werden (eine Zusammenstellung aller Stellen bei BERGER, 2005: 12). Für diese These spricht, dass Aristoteles selbst die Anatomai nie ohne Erwähnung der Historia Animalium zitiert. Das Verhältnis der beiden Werke wird an folgenden Beispielen besonders deutlich:

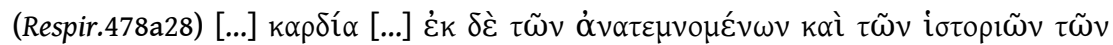

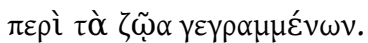

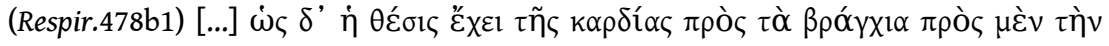

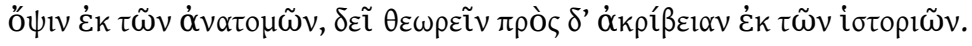

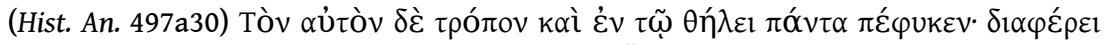

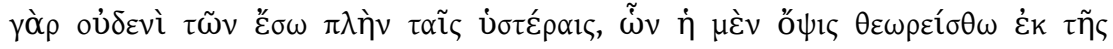

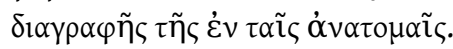

In der Epitome findet man Zitate aus allen Büchern der Historia Animalium, jedoch bezeichnenderweise keine Buchzählung? .

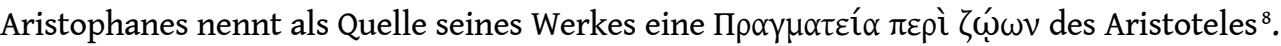
Dass es sich hierbei nicht nur um die Historia Animalium handelt, zeigt sich schon im ersten Buch der Epitome, in dem analog zum ersten Buch der Historia Animalium die Grundlagen gelegt, Begriffe geklärt und Phänomene behandelt werden, die alle Tiere betreffen. Es finden sich dementsprechend in den Kapiteln 2-26 des ersten Buches ein Überblick über die von Aristoteles verwendeten Fachtermini, in dem die über die Historia Animalium verstreuten Definitionen zusammengestellt sind, sowie eine Einteilung der Tiere nach äußeren Merkmalen und Lebensweisen. Es folgt in den Kapiteln 28-52 eine ausführliche Darstellung der Zeugung bei Vögeln, Insekten, Fischen und blutlosen Wassertieren, in der auch ein Kapitel der Urzeugung und den Tragzeiten der Tiere gewidmet ist ${ }^{9}$. Schließlich werden in den Kapiteln 54-97 Zeugung und Entwicklung im Mutterleib des Menschen behandelt. Dieser Abschnitt stützt sich nicht nur auf das siebte Buch der Historia Animalium, sondern vor allen Dingen auch auf De Generatione Animalium. Daneben werden auch andere Quellen herangezogen ${ }^{10}$, unter anderem De semine und De natura mulierum des Hippokrates oder eine unbekannte Quelle, die diese zitierte ${ }^{11}$.

Die Tierbeschreibungen in den Büchern II-IV der Epitome folgen einem genauen Schema, das im ersten Kapitel des zweiten Buches vorgegeben wird ${ }^{12}$ und sich folgendermaßen zusammenfassen lässt (DE STEFANI, 1904: 439; BERGER, 2005: 36; Hellmann, 2006).

Knappe Charakterisierung durch Gebiss, Fußform und Gang

Besonderheiten einzelner Körperteile

Beschreibung der inneren Teile

Paarung, Tragzeiten und Anzahl der Jungen, wie in der Hist. An. angegeben

Verschiedene Informationen meist aus dem achten Buch (IX) der Hist. An., häufig mit $\lambda \dot{\varepsilon} \gamma \varepsilon \tau \alpha \iota$ eingeleitet

Aufgrund dieses Schemas lassen sich Zitate aus der Epitome bei späteren Autoren gut identifizieren; so das folgende Beispiel aus Athenaios:

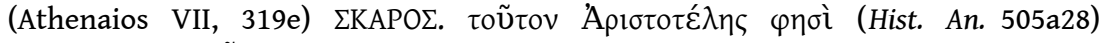

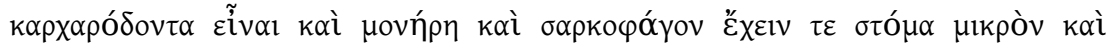




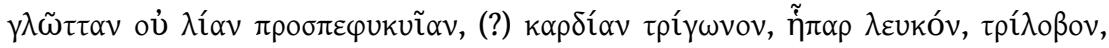

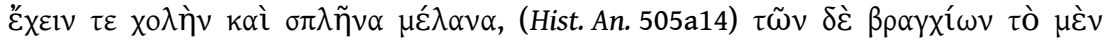

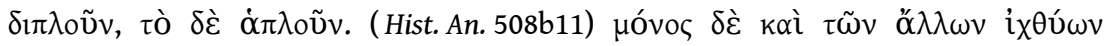

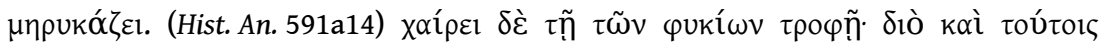

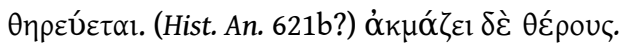

Aristophanes muss neben der Historia Animalium eine anatomische Schrift benutzt haben, in der er eine genaue Beschreibung der inneren Organe des Papageienfisches vorfand. Noch deutlicher wird dies bei der Beschreibung des Hundes, wie sie in der Sylloge überliefert ist:

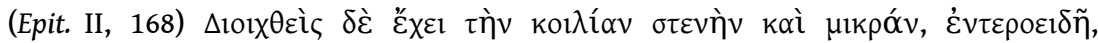

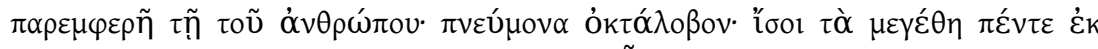

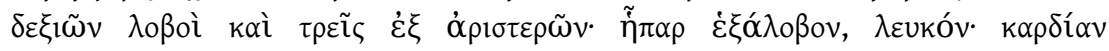

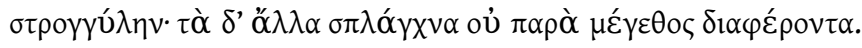

Ein Vergleich mit den anatomischen Angaben in den erhaltenen aristotelischen Tierschriften zeigt, dass Aristophanes diese Angaben nicht in den Anatomai des Aristoteles gefunden haben kann, sondern ein moderneres Werk benutzt haben muss: In der Historia Animalium geht Aristoteles für einige Tiere von zweiteiligen Lungen und Lebern aus (507a12-21). An anderer Stelle (506b29) beschreibt er die Niere des Rindes als viele kleine Nieren. In Part. An. spricht er von der Leber des Menschen und anderer

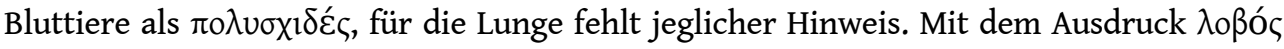

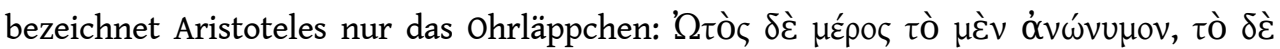

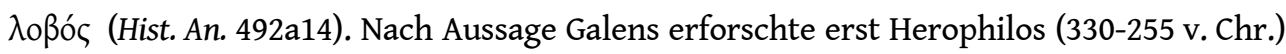
die inneren Organe genau und beschrieb dabei die Lappen der Organe:

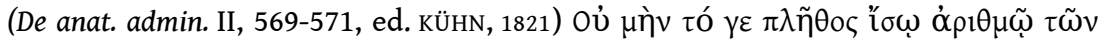

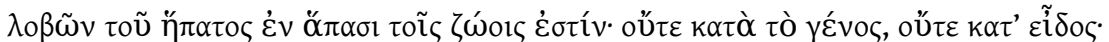

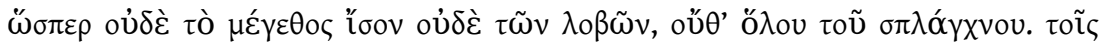

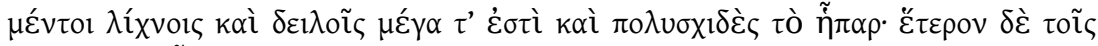

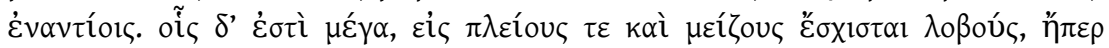

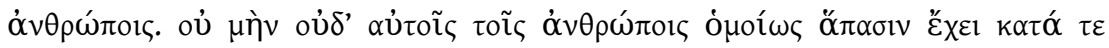

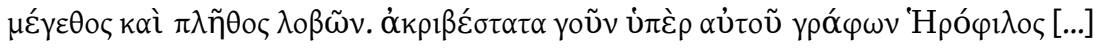

Nicht nur bei den anatomischen Besonderheiten, sondern auch bei der Auswahl der Tiere ging Aristophanes über das bei Aristoteles Gebotene hinaus: Die Beschreibungen von Maulwurf, Igel, Fledermaus und Mygale (Spitzmaus), die in der konstantinischen Sylloge überliefert sind,folgen dem aristophanischen Schema, so dass man sie der Epitome zuschreiben kann. Diese Tiere sind in den aristotelischen Tierschriften gar nicht oder nur knapp erwähnt. Hier könnte zum Beispiel ein verlorenes Werk des Theophrastos die

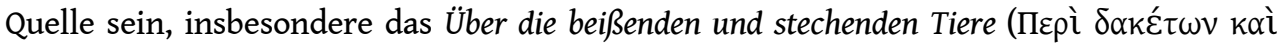
$\beta \lambda \eta \tau i k \tilde{v} v$ ) (solLenBERGER, 1984: 93, 122, 249). Erhaltene Parallelstellen zu den Texten der Epitome konnten nicht ermittelt werden, doch waren alle diese Tiere in antiker und byzantinischer Zeit als Bestandteile von Zaubermitteln und Heilmitteln beliebt; Maulwurf, Igel und Fledermaus werden etwa in den sogenannten Kyraniden ausführlich behandelt (KAIMAKES, 1976: 117-119, 137-138, 160), die Spitzmaus (Mygale) kommt im sogenannten Aelius Promotus (IHM, 1995: 58-59) vor, der bei ihr gerade die Art von Zähnen beschreibt, die in der Epitome der Fledermaus zugesprochen werden:

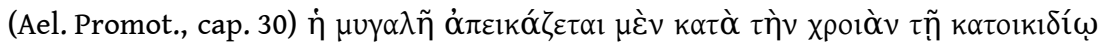

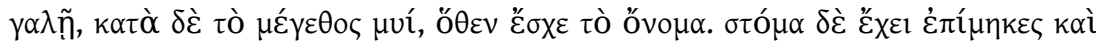

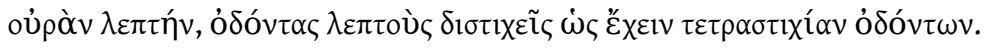

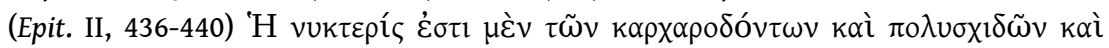

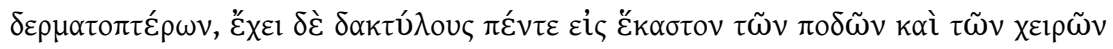




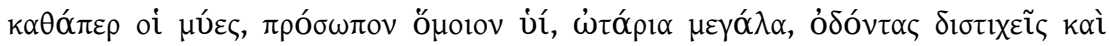

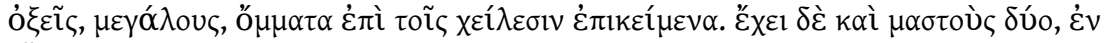

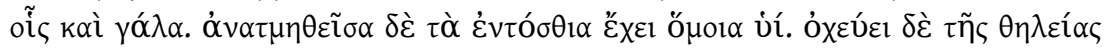

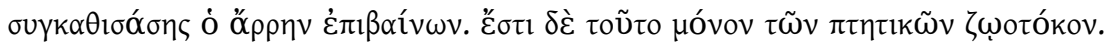

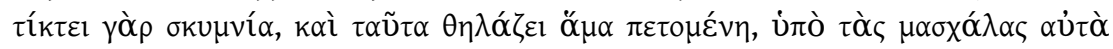

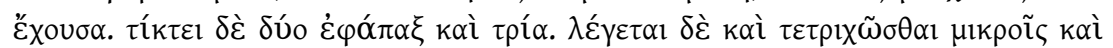

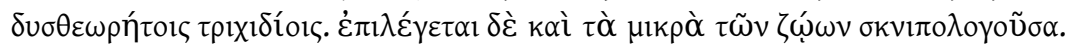

Es kann kein Zweifel daran bestehen, dass das Werk des Aristophanes keine bloße Epitome der Historia Animalium war, sondern im Einzelfall weiteres Wissen enthielt.

Daher stellt sich die Frage, ob der Autor seine Hauptquelle ergänzte oder ob er seine Informationen $\mathrm{zu}$ den einzelnen Tieren schon an anderer Stelle zusammengefasst vorfand. In diesem Zusammenhang richtete die Wissenschaft ihr besonderes Augenmerk auf die Zitate, für die Athenaios die aristotelischen Zoika als Quelle angibt (vgl. auch BERGER, 2005: 26-27). Handelt es sich hierbei um eine „interne Kartei“ des Peripatos (so DÜHRING, 1966: 513; DÜRING, 1950: 55-57), in der Tierbeschreibungen gesammelt wurden, ein verlorenes Werk des Aristoteles, oder sind die Zoika gar identisch mit der Epitome des Aristophanes (KULLMANN, 1998, 512 mit der älteren Literatur)?

\section{Epitome und Zoika}

In den um 190 nach Christus verfassten Deipnosophistai(ed. KAIBEL, 1887-1890) schildert Athenaios die Unterhaltung mehrerer gelehrter Männer über viele Themen, insbesondere auch über die ihnen aufgetischten Speisen. Dabei werden unter dem Namen des Aristoteles verschiedene Werke zitiert (vgl. die Zusammenstellung aller Parallelstellen bei DÜRING, 1950: 41-47; GIGON, 1987: Fr. 180-268). Stehen unter einem Lemma Texte aus mehreren von ihnen, wird einige Male eines mit $\dot{\varepsilon} v$ ö $\lambda \lambda_{0}$ เ bezeichnet (vgl. Athenaios,

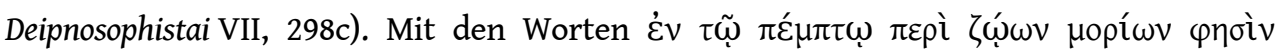

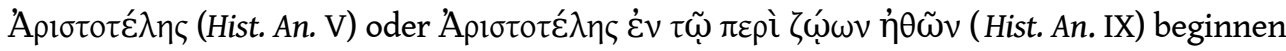
Zitate, die den Text der Historia Animalium exzerpierend, aber wörtlich wiedergeben.Ein

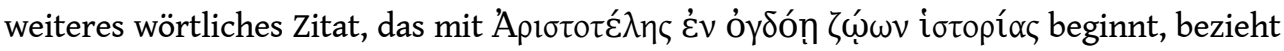
sich auf Vögel. Es stammt möglicherweise aus einem Vogelbuch, von der Art wie es Alexandros von Myndos im 1. Jh. n. Chr. verfasste (vgl. BERGER, 2005: 38-39). Texte, die mit

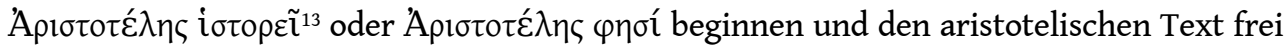
wiedergeben, sind der Epitome des Aristophanes entnommen. Zitate, als deren Quelle ćv

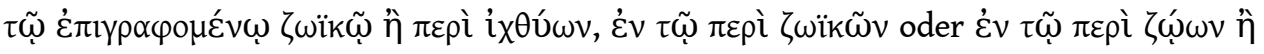
i $\chi \theta \dot{v} \omega v$ angegeben wird, stammen nicht aus den aristotelischen Tierschriften und lassen sich auch nicht mit den erhaltenen Texten des Aristophanes vergleichen. Sie enthalten Tierbeschreibungen, in denen das Äußere der Tiere in einer Art und Weise genau dargestellt wird, wie es bei Aristoteles nie vorkommt. Er interessierte sich zwar für gemeinsame Merkmale von Tieren, eine Aussage wie „alle Tiere mit schwarzen Punkten“ hatte jedoch in seinem System keinen Platz.

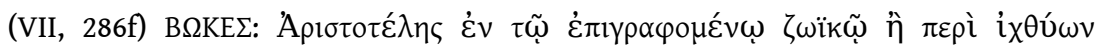

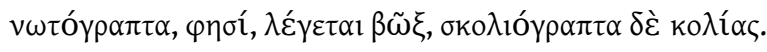

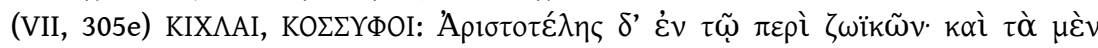

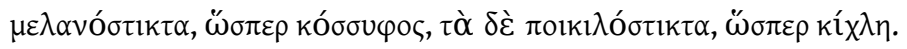

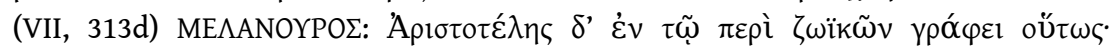

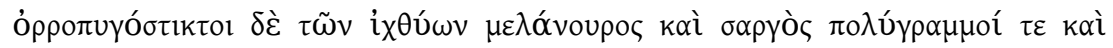
$\mu \varepsilon \lambda \alpha v o ́ \gamma \rho \alpha \mu \mu о$ l. 


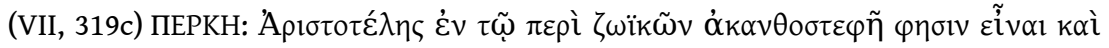

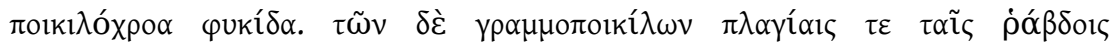

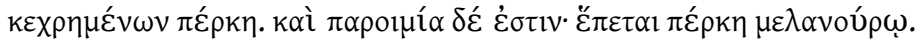

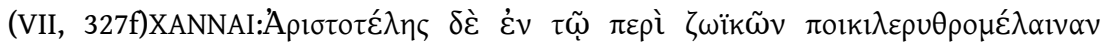

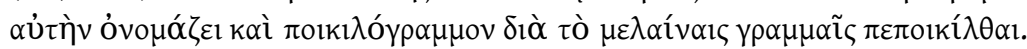
benutzt hat.

In den folgenden Lemmata wird ausdrücklich auf Unterschiede zwischen den Aussagen

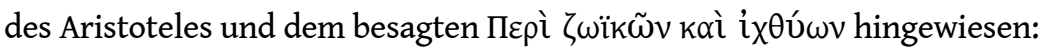

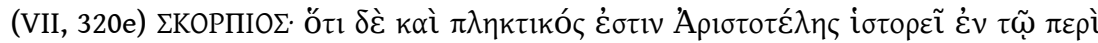

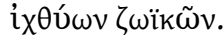

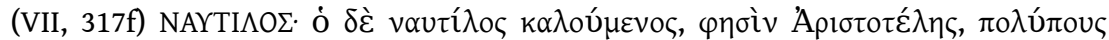

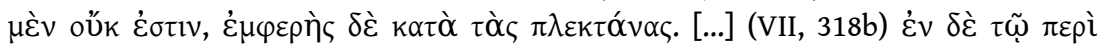

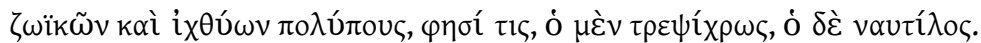

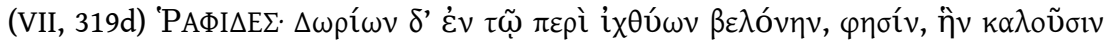

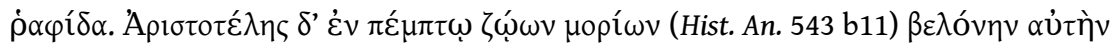

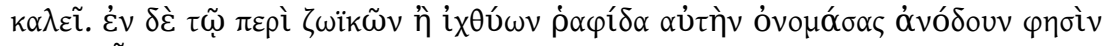

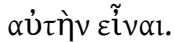

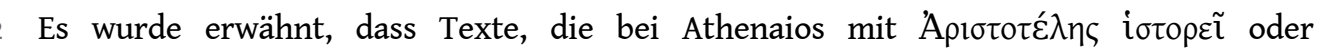

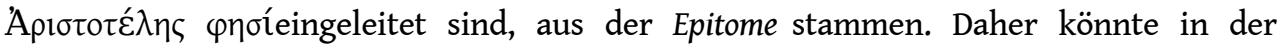
Beschreibung des Skorpions die Epitome die Quelle für das Zitat aus den Zoika sein; in der Beschreibung des Nautilus dagegen scheint ein Aristoteles-Zitat aus der Epitome zu stammen, dessen Inhalt nicht mit den Zoika übereinstimmt. Es ist daher nicht möglich, dass Epitome und Zoika identisch sind, wohl aber denkbar, dass Aristophanes die Zoika

Die Unterschiede zwischen beiden Werken werden auch in den Lemmata deutlich, die sich mit dem ersten Buch der Epitome in Verbindung bringen lassen, etwa die Beschreibung der Scholle $(\psi \tilde{\eta} \tau \tau \alpha)$. Athenaios zitiert mit Diokles zunächst ein medizinisches Handbuch, dann ein Stück aus dem zweiten Buch der Homoia des Speusippos, der schon vor Aristoteles eine Systematik entwickelte. Es folgt ein Zitat aus der Historia Animalium, ein Zitat aus den Zoika und abschließend ein kurzes Stück aus dem Fischbuch des Dorion, der ins erste vorchristliche Jahrhundert gesetzt wird.

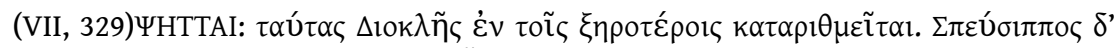

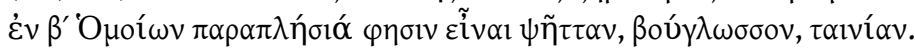

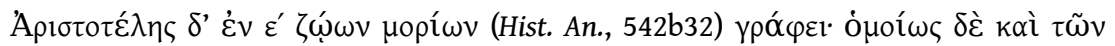

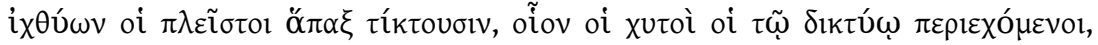

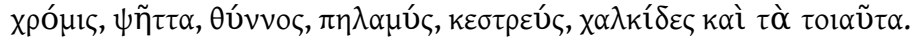

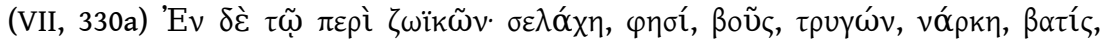

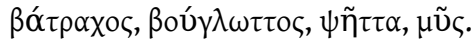

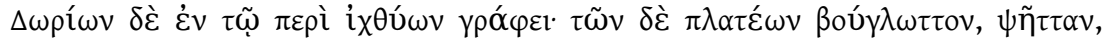

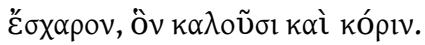

Während das Zitat aus der Historia Animalium wörtlich wiedergegeben ist, ist das Zitat aus den Zoika an anderer Stelle nicht zu belegen. Es wurde zunächst sicher $\mu \tilde{v} \varsigma$ mit $\pi \eta \lambda \alpha \mu$ v́

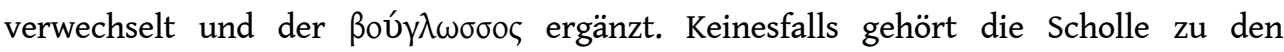

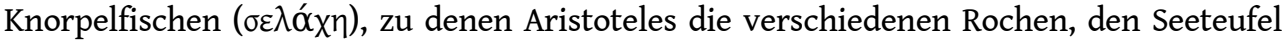

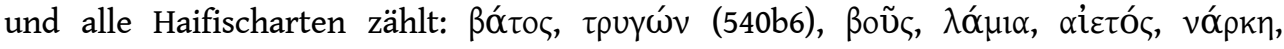

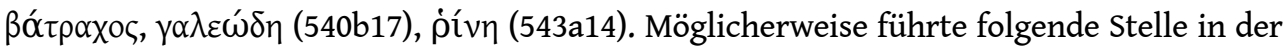
Historia Animalium, in der die Scholle neben ihnen erwähnt wird, zu dieser Verwirrung:

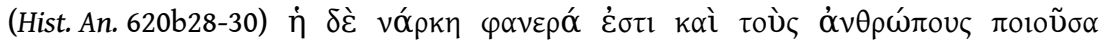

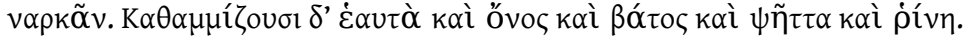


Vergleichen wir nun den entsprechenden Text in der Epitome des Aristophanes, zeigt sich, dass Aristophanes die Scholle nicht unter die Knorpelfische zählt. Allerdings definiert er diese auch anders als Aristoteles. Für ihn sind das alle Fische, die keine Schuppen haben, unter ihnen auch die Seeungeheuer sowie Aal und Muräne. Es werden zwar vó $\rho \kappa \eta$,

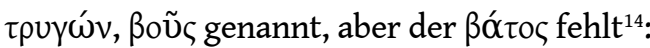

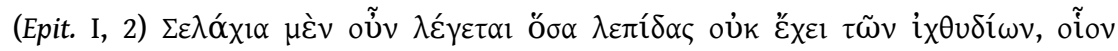

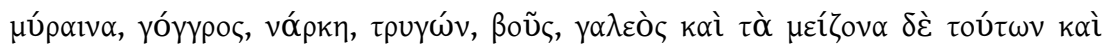

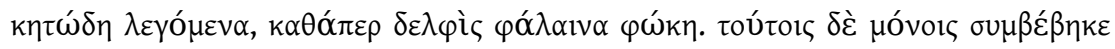
$\tau \tilde{\omega} v \dot{\varepsilon} v u ́ \delta \rho \omega \nu \zeta \omega$

Seine Definition der Knorpelfische stammt wohl aus der folgenden Stelle der Historia Animalium, wo die Seeungeheuer gemeinsam mit den Knorpelfischen behandelt werden:

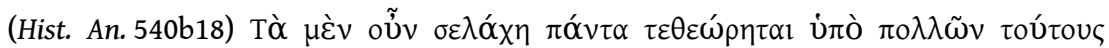

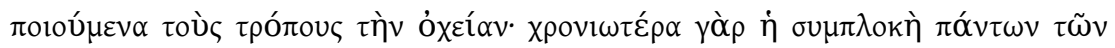

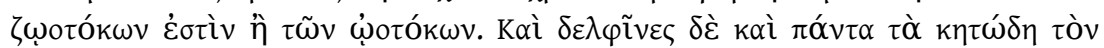

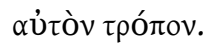

Ähnliches ist in folgendem Beispiel $\mathrm{zu}$ beobachten, in dem es um Weichtiere und Polypoden geht. Athenaios schreibt:

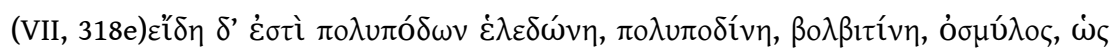

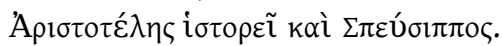

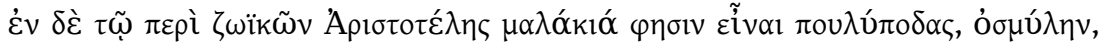

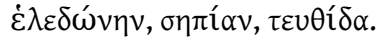

Vergleichen wir zunächst die entsprechenden Stellen in der Historia Animalium:

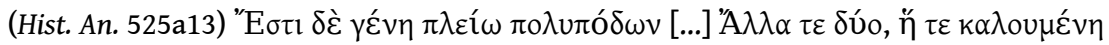

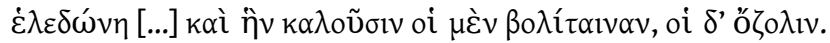

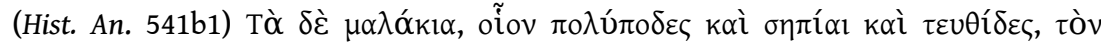

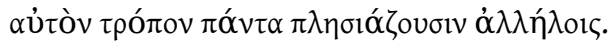

9 Wenn man eine gewisse Schwankungsbreite bei den Tiernamen in Kauf nimmt, widersprechen sich die Texte nicht: Bei $\beta \circ \lambda \beta \imath \tau i ́ v \eta ~ \beta o \lambda i ́ \tau \alpha ı v \alpha$ handelt es sich sicher um denselben Fisch, der an anderer Stelle als $\beta$ o $\lambda \beta$ otív $\eta$ bezeichnet wird. Der bei Athenaios

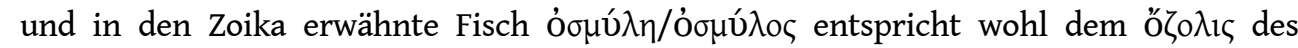
Aristoteles, wie eine Stelle aus den Chiliaden des Tzetzes zeigt (ed. LEONE, 1968: Chilias 5, Hist. 25):

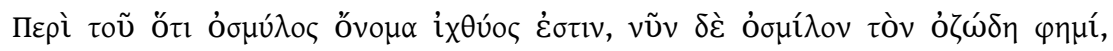

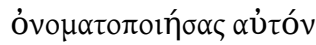

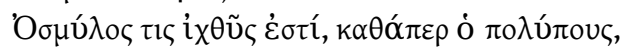

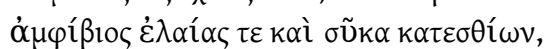

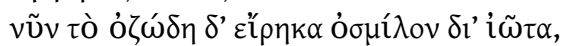

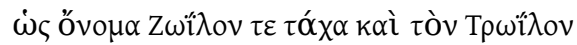

Es liegt hier eine klassische Majuskelverlesung und eine itazistische Verschreibung vor: OZ $\triangle \mathrm{HN}$ wird zu OZO $\Lambda$ IN. Dass die Namen der Fische schwanken, zeigt die folgende Stelle aus Pollux (ed. BETHE, 1931):

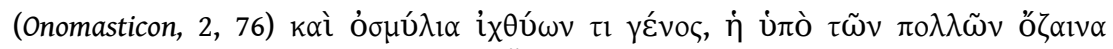

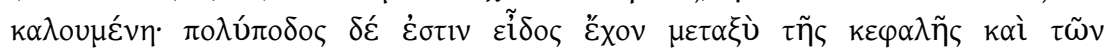

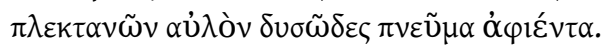

Ausgeschlossen erscheint aber, dass der Text der Zoika auf die Epitome zurückgeht, denn dort heißt es: 


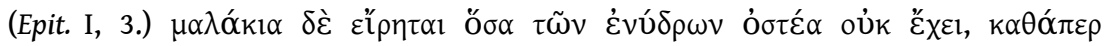

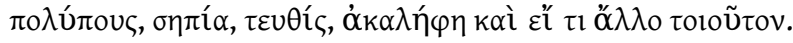

Der Text der Epitome ist gesichert, da gerade diese Definition von Ailianos (ed. SCHOLFIELD, 1959: XI, 37) und in den Oppian-Scholien überliefert wird (ed. BUSSEMAKER, 1849: Scholion 638). Aristophanes hat hier ähnlich eigenmächtig entschieden wie bei den Knorpelfischen, denn die von ihm unter die Weichtiere gezählte Seenadel ( $\alpha \kappa \alpha \lambda \eta ́ \varphi \eta)$ wird bei Aristoteles in De Partibus Animalium ausdrücklich keinem Genus zugeordnet:

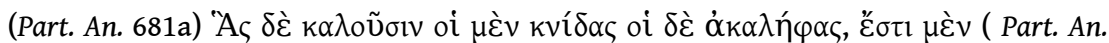

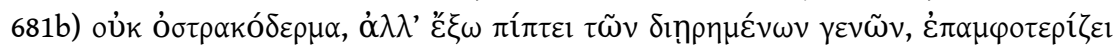

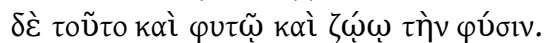

Fassen wir zusammen: Aristophanes benutzte neben den aristotelischen Tierschriften weitere Quellen; darunter befand sich eine modernere Darstellung der Anatomie, wie sie etwa Herophilos zugeschrieben wird. Man kann auch davon ausgehen, dass er die Werke des Theophrastos kannte und möglicherweise ein Buch, das bei Athenaios mit $\dot{\varepsilon} v \tau \tilde{\omega}$

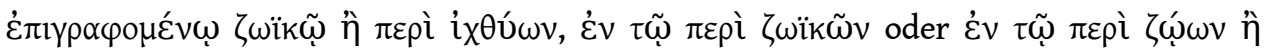
i $\chi \theta u ́ \omega v$ bezeichnet wird. Dieses Werk, das in der modernen Sekundärliteratur als Zoika auftaucht, ist sicher nicht identisch mit der Epitome des Aristophanes, sondern war wahrscheinlich ein Buch über Fische, wie es von Athenaios zum Beispiel dem AristotelesSchüler Klearchos zugeschrieben wird:

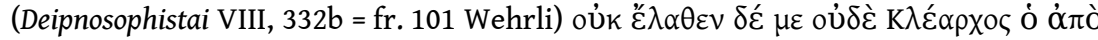

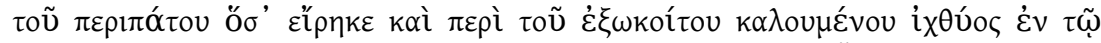

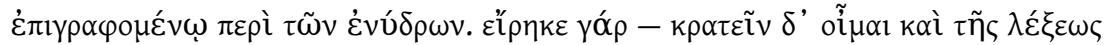

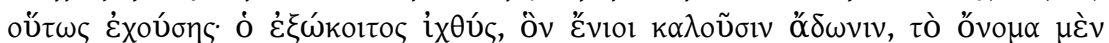

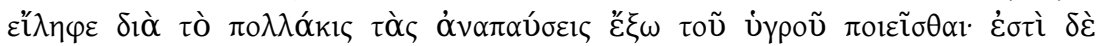

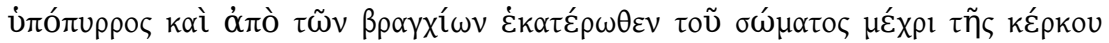

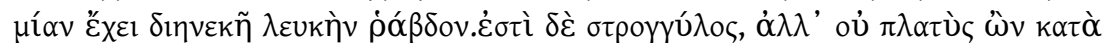

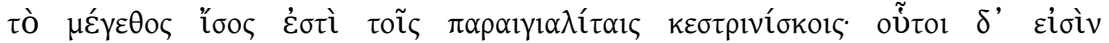

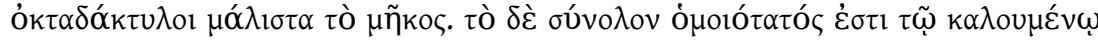

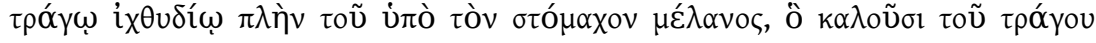
$\pi \omega ́ \gamma \omega \vee \alpha$.

\section{BIBLIOGRAPHIE}

ATHENAIOS siehe KAIBEL, 1887-1890.

BALme David M. (ed.), Aristotle, Historia Animalium, I: Text, Cambridge : University Press, 2002.

BERGER Friederike, Die Textgeschichte der Historia Animalium des Aristoteles, Wiesbaden :

Reichert, 2005, « Serta Graeca, 21 ».

BETHE Erich (ed.), Pollucis onomasticon, II, Leipzig: Teubner, 1931, « Lexicographi Graeci, 9.2 ».

BEULlENS Pieter, BossIER Fernand (edd.), De Historia Animalium, Translatio Guillelmi de Morbeka, I: Lib. I-V, Leiden - Boston - Köln: Brill, 2000, « Aristoteles Latinus, XVII/2.I.1 ». 
BUSSEMAKER Ulco Cats (ed.) Scholia et Eutecnii paraphrasis in Nicandrum, Scholia et paraphrasis in Oppianum... , Paris : Didot, 1849, S. 260-364.

Cuomo Valentina, « Athos Dionysiou 180 + Paris. Suppl. Grec. 495: Un nuovo manoscritto di Teodosio Principe », BZ, 98, 2005, p. 23-34.

De Stefani Luigi, «Per l' epitome de animalibus di Aristofane di Bizanzio », Studi Italiani di Filologia

Classica, 12, 1904, p. 421-445.

DE STEFANI Luigi, « Un'epitome Laurenziana della Sylloge Constantini de natura animalium », Studi Italiani di Filologia Classica, 20, 1913, p. 189-203.

DÜRING Ingemar, Aristotle in the Ancient Biographical Tradition, Göteborg: Almqvist \& Wiksell, 1957, « Studia Graeca et Latina Gothoburgensia, 5 ».

EPIT(OME) siehe LAMBROS, 1885.

GIGON Olaf (ed.), Aristotelis Opera, III: Librorum deperditorum fragmenta, Berlin: De Gruyter, 1987.

HELLMANN Oliver, “ „Multimedia“ im Lykeion? Zu Funktionen der Anatomai in der aristotelischen Biologie », Antike Naturwissenschaft und ihre Rezeption, 14, 2004, p. 65-86.

HELLMANN Oliver, «Peripatetic Biology and the Epitome of Aristophanes of Byzantium », in: ForTenbaugh W. W., White S. A., Aristo of Ceos, New Brunswick: Transaction Publishers 2006, p. 329-373.

HENRY René, Photius, Bibliothèque, II: Codices 84-185, Paris: Les Belles Lettres, 1960.

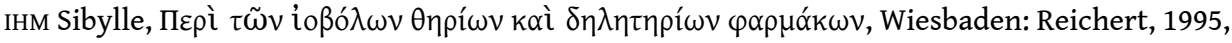
«Serta Graeca, 4 ».

KAIMAKES Dimitris V., Die Kyraniden, Meisenheim am Glan: Hain, 1976, « Beiträge zur klassischen Philologie, 76 ".

KAIBEL Georg, Athenaei Naucratitae Dipnosophistarum libri I-XV, I-III, Leipzig : Teubner, 1887-1890.

KÜHN Karl Gottlob(ed.), Claudii Galeni Opera omnia, II, Leipzig : Cnobloch, 1821.

KULLMANN Wolfgang, «Zoologische Sammelwerke in der Antike », in KULLMANN W., ALTHOFF J. et ASPER M., Gattungen wissenschaftlicher Literatur in der Antike, Tübingen: Narr, 1998, p. 121-139.

LAMBRos Spyridon (ed.), Excerptorum Constantini de Natura Animalium Epitome subiunctis Aeliani Timothei aliorumque Eclogis, Berlin: Reimer, 1885, « Supplementum Aristotelicum, 1 ».

LEONE Pietro Luigi M., Ioannis Tzetzae historiae. Neapel: Libreria Scientifica Editrice, 1968.

MARCovich Miroslav (ed.), Diogenis Laertii Vitae philosophorvm, I: Libri I-X, Stuttgart [u. a.]: Teubner, 1999.

MONDRAIN Brigitte, « La constitution de corpus d'Aristote et de ses commentateurs aux XIII ${ }^{\mathrm{e}}-\mathrm{XIV}^{\mathrm{e}}$ siècles ", Codices Manuscripti, 29, 2000, p. 11-33.

MORAUX Paul, Der Aristotelismus bei den Griechen von Andronikos bis Alexander von Aphrodisias, I: Die Renaissance des Aristotelismus im 1. Jh. v. Chr., Berlin : de Gruyter, 1973.

MUSso Olympius(ed.), [Antigonus Carystius], Rerum mirabilium collectio, Neapel : Bibliopolis, 1985.

PAPPA Eleni(ed.), Georgios Pachymeres, Philosophia. Liber VI: In Aristotelis De partibus animalium commentarium, Athen: Academy of Athens, 2009, « Corpus philosophorum medii aevi.

Commentaria in Aristotelem Byzantina IV/1 ». 
ROSE Valentin, Anecdota Graeca et Graecolatina II, Berlin: Dümmler, 1870.

SCHOLFIELD Alwyn F. (ed.), Aelian, On the characteristics of animals, II, London: Heinemann, 1959.

SOLLENBERGER Michael. G., Diogenes Laertius' Life of Theophrastus. A crit. ed. of the Greek text with a transl. and commentary, Diss. Rutgers University (New Jersey), Ann Arbor - New Brunswick: Univ. Microfilms International, 1984.

STÜCKELBERGER Alfred, Bild und Wort, Mainz: Von Zabern, 1994.

STÜCKELBERGER Alfred, « Aristoteles illustratus », Museum Helveticum, 50, 1993, p. 131-143.

VUILLEMIN-DIEM Gudrun, « Untersuchungen zu Wilhelm von Moerbekes Metaphysikübersetzung », in : ZIMMERMANN A., Studien zur mittelalterlichen Geistesgeschichte und ihren Quellen, Berlin: De Gruyter, 1982, « Miscellanea mediaevalia, 15 », p. 102-172, 208.

\section{NOTES}

1. Bei Diogenes Laertios ist schon für Theophrastos eine Epitome in 6 Büchern überliefert, über die wir aber sonst nichts wissen (vgl. weiterführende Literatur bei BERGER, 2005: 23).

2. Der folgende Beitrag beruht zu großen Teilen auf meinem Buch „Die Textgeschichte der Historia Animalium des Aristoteles“ (BERGER, 2005).

3. BrLibr inv. 2242 (2./3. Jh.) überliefert fragmentarisch Epit. II, 169-177 (Corpus dei papiri filosofici greci e latini, I, 1989, 36T).

4. Siehe dazu unten die Quellen der Epitome.

5. cuомо, 2005, erwähnt folgende Handschriften: Paris. suppl. gr. 495: Buch I. - Athos Dionysiu 180: Buch II. - Laur. 86, 8 (15. Jh.): Exzerpte aus Buch I und II. - Paris. gr. 1921 (14. Jh.): Buch I, 1-22; ed. LAMPRos, VI-VIII. - Athos Iviron 388 (16. Jh.): Buch I, 1-22. - Vat. gr. 93 (14. Jh.): Buch I, 2-6, 9, 7, 8, 10-18.- Ricc. gr. 12 (15. Jh.): Buch I, 2-6.

6. Vgl. MORAUX, 1973, 60-63 zur Tendenz, Traktate zu größeren Einheiten zusammenzufassen.

7. Ein vermeintliches Zitat aus dem siebten Buch (Hist. An. 604a; Buch VIII in den Ausgaben) ist

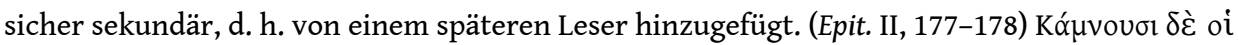

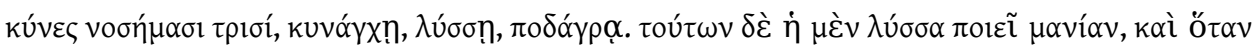

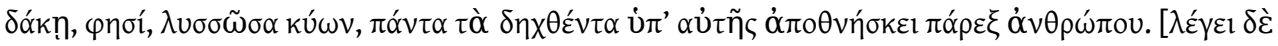

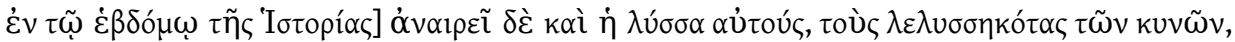

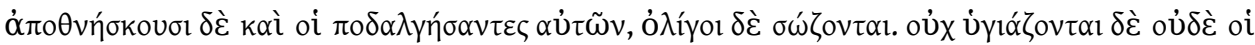

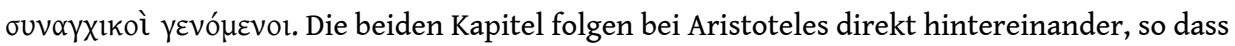
kein Grund vorhanden ist, die Historia Animalium hier besonders zu erwähnen; auch leitet

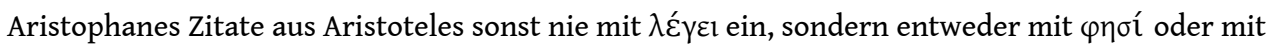

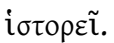

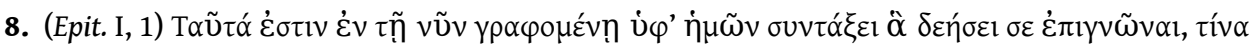

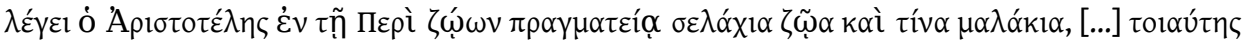

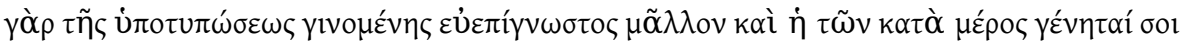

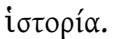

9. Das Kapitel 35 über die Bienen stammt nicht aus der Historia Animalium.

10. Vgl. Epit. I, 69 (= Hippokrates VII, De nat. mul. 95,4), 73 (= De nat. mul. 32, 165; De sem. 54, 16), 74 (= Hippokrates IX, De mul. affect. 17,7), 88 (= ?), II, 30-36 (= ?).

11. Die letzten Kapitel des ersten Buches gehören nicht zum Textbestand der Epitome, es handelt sich vielmehr um eine Zusammenstellung von einzigartigen Besonderheiten verschiedener Lebewesen, die aus vielen verschiedenen aristotelischen Werken stammt und eindeutig in 
paradoxographischer Tradition steht. Eine ähnliche Auswahl, die unter dem Namen des Antigonos von Karystosüberliefert ist, wird ins 10. Jh. n. Chr. datiert (ed. MUSSO, 1985).

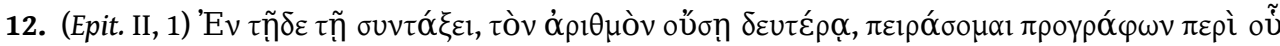

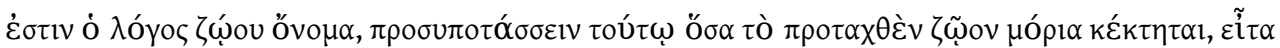

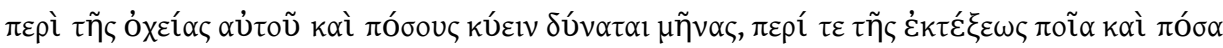
ن்

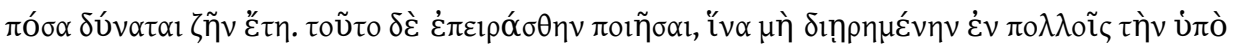

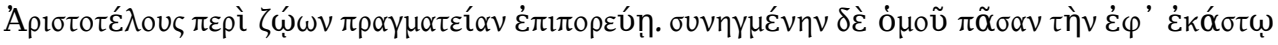
$\zeta \omega$ ¡

13. So wird auch in der Epitome (II, 215) ein wörtliches Zitat eingeleitet.

14. Dies könnte die Quelle für folgendes Lemma bei Athenaios sein: (VII, 26) BATI $\Sigma$. BATPAXO $\Sigma$.

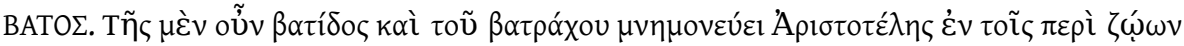

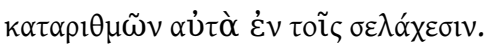

\section{RÉSUMÉS}

Aristophanes von Byzanz zog für seine Epitome neben der Historia Animalium noch weitere Quellen heran, die heute verloren sind, etwa die zoologischen Werke des Theophrastos. Seine anatomischen Kenntnisse bezog Aristophanes nicht aus den Anatomai des Aristoteles, sondern aus einem moderneren Werk. Die Epitome ist keinesfalls identisch mit den bei Athenaios dem Aristoteles zugeschriebenen Zoika. Die Tatsache, dass Aristophanes die aristotelischen Tierschriften vor ihrer Edition durch Andronikos von Rhodos benutzte, macht sein Werk für die Textgeschichte der Historia Animalium interessant.

Aristophanes of Byzantium consulted for his Epitome in addition to the Historia Animalium, also other sources which are now lost, such as the zoological works of Theophrastus. Aristophanes drew his knowledge of anatomy not from Aristotle's Anatomai, but from a more recent work. The Epitome is not identical to the Zoika attributed to Aristotle by Athenaeus. The fact, that Aristophanes of Byzantium used Aristotle's writings on animals before their edition by Andronicus of Rhodes makes his work important for the textual history of the Historia Animalium.

\section{INDEX}

Keywords : Aristotle, Athenaeus, Aristophanes Byzantius, Historia Animalium, History of Text Schlüsselwörter : Aristoteles, Athenaios, Aristophanes von Byzanz, Historia Animalium, History of Text 


\section{AUTEUR}

\section{FRIEDERIKE BERGER}

Bayerische Staatsbibliothek, Abteilung Handschriften und Alte Drucke

Ludwigstr. 16 - D-80539 München

Berger@bsb-muenchen.de

Friederike Berger studied Classics and Byzantine Philology in Munich, Thessaloniki and Berlin (M.A. 1987; Dr.Phil. 2000), and Library and Information Studies in Berlin (M.A. 2002). From 2004 to 2012 she was a member of the Department of Manuscripts and Early Printed Books at the Bavarian State Library in Munich, since April 2012 she is working on a catalogue of the Greek manuscripts at the University Library of Leipzig.

Du même auteur : BERGER Friederike, « Bemerkungen zur Überlieferungsgeschichte der aristotelischen Schrift De Incessu Animalium », in : BERGER F. et BROCKMANN Ch., Symbolae Berolinenses für Dieter Harlfinger, Amsterdam : Hakkert, 1993, p. 23-42. 